\title{
Disparités géographiques de fécondité et de nuptialité en Tunisie : divergences et convergences'
}

\author{
Zahia Ouadah-Bedidi ${ }^{1}$, Jacques Vallin ${ }^{2}$ et lbtihel Bouchoucha ${ }^{3}$ \\ 1URMIS, Université de Paris Diderot et INED ; ${ }^{2}$ INED ; ${ }^{3}$ Université de Montréal \\ nathsawadogo@gmail.com
}

\section{Résumé}

De 1965 à 1999, la fécondité tunisienne est tombée de 7,5 à 2,I enfants par femme et s'est ensuite maintenue à ce niveau pendant plus de dix ans. De son côté, l'âge moyen au premier mariage des femmes, dont la forte augmentation a été un facteur important de la maitrise de la fécondité, s'est stabilisé dès la fin des années 1980. Passé de 21 à 29 ans, il n'a guère bougé depuis. Ces faits sont connus au niveau national, mais leur diversité géographique l'est beaucoup moins. Pour analyser cette dernière, plusieurs sources de données, souvent difficiles d'accès, ont été mobilisées. Elles nous permettent de voir que tant du côté de la fécondité que de celui de la nuptialité, la géographie a beaucoup changé. Non seulement partout la fécondité a profondément baissé tandis que l'âge moyen au premier mariage des femmes augmentait très fortement, mais les disparités régionales ont beaucoup évolué. Après une phase de forte divergence, les fécondités régionales ont convergé vers le taux de remplacement. Quant à la carte de l'âge au mariage, elle s'est complètement inversée. Il en résulte que les rapports entre la diversité régionale de la fécondité et celle de la nuptialité ne sont ni simples ni très affirmés.

Mots-clés : Tunisie, fécondité, taux de fécondité totale, nuptialité, âge moyen au premier mariage, disparités géographiques

\begin{abstract}
From 1965 to 1999, Tunisian fertility fell from 7,5 children per women to 2,I, and then kept that replacement level for more than 10 years before rising slightly. On its side, female mean age at marriage, the huge increase of which (from 21 to 29 years) was a prominent factor of fertility decrease, stabilized at the end of the 1980s. Facts are well known at the national level but to what extent national means result from contrasted changes at the regional one remains quite unclear. To analyze geographical disparities, various sources, often difficult to access, were mobilized. They allowed seeing that for both fertility and nuptiality geography changed a lot. Not only fertility fell down deeply while women age at first marriage increased a lot everywhere, but regional diversity changed dramatically. After a step of steady divergence regional fertilities converged towards the replacement level almost perfectly. Concerning the map of age at marriage, it reversed completely. As a consequence, the relations between regional diversities of fertility and nuptiality are neither simple nor well asserted.
\end{abstract}

Keywords: Tunisia, fertility, total fertility rates, nuptiality, mean age at first marriage, geographical diversity

\section{Introduction}

Au lendemain de l'indépendance de la Tunisie (1956), la fécondité était très élevée : plus de 7,5 enfants par femme (Waltisperger et al., 200I). Elle s'est maintenue à ce niveau à peu près constamment dans les dix années qui ont suivi, à l'exception d'un pic assez remarquable, en 1964, lié à l'annonce d'une loi sur l'âge au mariageii. Plus que le programme de planning familial encore balbutiant à cette époque, cette loi de 1964 a été le symbole du facteur déclenchant de la baisse vertigineuse de la fécondité tunisienne qui est tombée de 7,5 enfants par femme en 1965 à 2,I en 1999. Depuis, de façon tout aussi remarquable, la fécondité s'est stabilisée pour une bonne dizaine d'années à ce niveau de 2,I enfants par femme qui assure exactement le remplacement des générations. Ces dernières années, la fécondité semble toutefois avoir sensiblement raugmenté. D'après une récente étude de l'INS elle aurait même atteint 2,4 enfants par femme en 2014 (INS, 20I5) iii. Cette remontée pourrait être liée à un certain retour de la société tunisienne à des valeurs traditionnelles mais elle pourrait tout aussi bien s'expliquer mécaniquement par l'arrêt de la montée, elle aussi 
vertigineuse, de l'âge moyen au mariage qui a caractérisé les décennies précédentes.

De 1965 à 1999, les taux de fécondité par groupe d'âges ont systématiquement baissé à tous les âges. La baisse a été particulièrement profonde pour les taux à 20-24 et 25-29 ans. Pour les années récentes, le retournement à la hausse porte surtout sur les taux à 30-34 ans et 35-39 ans alors que le taux à 20-24 ans continue à diminuer. Quant à la période de relative stabilité de la fécondité totale (|999-20|2), elle résulte en fait d'une compensation entre des groupes d'âge où la fécondité avait commencé à raugmenter et d'autres où elle continuait à diminuer. Le contraste a été particulièrement grand entre les taux à 20-24 et 35-39 ans dont les trajectoires se sont croisées dans les années 1990. Ces variations de la structure par âge de la fécondité laissent à penser que des variations de même nature pouraient avoir lieu entre entités géographiques, sujet presque totalement ignoré de la littérature en ce qui concerne la Tunisie.

On sait par ailleurs qu'en Tunisie, comme dans les trois autres pays du Maghreb (Algérie, Libye, Maroc), l'élévation spectaculaire de l'âge au premier mariage des femmes a été le premier (et plus ou moins longtemps le principal) facteur de la baisse de la fécondité, la diffusion de la contraception n'étant venu prendre le relais que plus tard (Ouadah-Bedidi et al., 2000, 20/2). On peut donc se demander si des variations géographiques de la nuptialité pourraient être mises en relation avec celles de la fécondité ?

Nous nous intéresserons donc ici à deux questions. D'une part retrouve-t-on au niveau régional les mêmes évolutions de fécondité que celles observées au niveau national ? Et, d'autre part, si, comme il est probable, les comportements féconds ont varié d'une région à l'autre, ces variations ont-elles un rapport avec celles que l'on pourrait aussi observer pour la nuptialité, dont on sait qu'elle a joué un rôle majeur au niveau national ?

Après une brève revue de la littérature et une présentation des données et méthodes, nous analyserons la géographie de la fécondité tunisienne et son évolution et répéterons le même exercice pour la nuptialité pour finalement explorer les liens existant entre ces deux phénomènes.

\section{Revue de la littérature}

Les études sur la baisse de la fécondité tunisienne sont abondantes, au niveau national. Dès la fin des années 1960, de premières études ont tenté de mesurer le rôle que pourrait jouer la mise en place du programme de planning familial (Vallin et Lapham 1969, Lapham 1970), soulignant aussi, dès cette époque l'influence des comportement matrimoniaux. Dans les années 1970 et 1980, l'accent a davantage été mis sur la diffucion de la contraception (Seklani
1974 ; Jemai et Jemai 1979, I982 ; Ayad et Zoughlami 1985; Ayad et Jemai 1991). Cependant, dès les années 1980 (Tabutin, 1982) mais surtout à partir des années 1990 on a remis l'accent sur le rôle joué par l'élévation de l'âge au mariage dans la baisse de la fécondité (Adlakha et al. 1991). Le rôle majeur joué par l'évolution des comportements matrimoniaux dans la baisse de la fécondité $a$, en réalité, été un phénomène très typiquement maghrébin, lié au fait que, contrairement aux évolutions observées dans la plupart des pays du Sud, l'élévation de l'âge au mariage y a atteint des records rarement égalés (Ayed et Jemai 200I, Bourchachen 1995, Ouadah-Bedidi 2004).

Les études sur la géographie de la fécondité en Tunisie sont extêmement rares et généralement très succintes sur la base d'indicateurs très sommaires (Bouraoui 1992, Ben Jelassi 1992), quand elles ne se limitent pas à quelques pragraphes dans une description générale de la fécondité (Sandron, 1998). L'étude des variations régionales de la nuptialité est encore plus rare. Celle de Lilia Ben Salem et Thérèse Locoh (200I) s'efforce néanmoins de mesurer l'évolution de la struture régionale de l'âge au premier mariage des femmes entre les recensements de 1966 et 1994, en relation avec le contexte plus général de transformation de la famille tunisienne. Le problème reste que cette étude ne repose que sur des estimations par la méthode de Hajnal (1953) qui ne permet pas de dater correctement les observations faites.

Concernant, enfin, les relations entre la géographie tunisienne de la fécondité et celle de la nuptialité, nous n'en avons trouvé aucune trace dans la littérature.

\section{Données et méthodes}

Du point de vue administratif, la Tunisie est divisée en gouvernorats, eux-mêmes subdivisés en délégations. À l'échelon suivant, on trouve les secteurs (anciens cheihkhats) en milieu rural et les communes en milieu urbain. En tout état de cause, compte tenu des données accessibles, cette étude doit se limiter au niveau des gouvernorats.

\section{Données disponibles sur les naissances et les mariages}

La Tunisie dispose d'un système d'enregistrement des faits d'état civil qui fonctionne assez bien depuis les années 1950, sous la responsabilité des chefs de secteur dans les secteurs et celle des maires dans les communes. Les données sont largement informatisées, parfois même dès le moment de l'enregistrement des événements. On devrait donc pouvoir disposer d'une information très riche sur les mariages et les naissances. 
La statistique des naissances par groupe d'âge de la mère n'est cependant guère accessible qu'au niveau des gouvernaorats, surtout pour les années les plus anciennes pour lesquelles il faut se contenter de leur publication, plutôt irrégulière, dans les annuaires statistiques. II est difficile de remonter le temps avant le début des années 1970. Et même, pour la période postérieure à 1970, nous n'avons pas pu retrouver la trace de données sur les naissances selon l'âge de la mère et le gouvernorat pour les années 1972, 1973, 1983, 1985, 1986, 1989 et 1990. Les statistiques de naissances par gouvernorat sont dressées séparément en fonction du lieu naissance et en fonction du lieu de domicile de la mère, ce qui est très important non seulement pour les accouchements en maternité mais aussi pour les accouchements au domicile parental de la mère.

La statistique par gouvernorat des mariages enregistrés à l'état civil (selon l'âge, le sexe et l'état matrimonial antérieur des époux) est encore moins facilement accessible que celle des naissances. C'est la raison pour laquelle les rares études sur la question ne reposent que sur des âges moyens au premier mariage estimés par la méthode de Hajnal.

\section{Estimations de population}

Depuis son indépendance, la Tunisie a tenu 7 recensements : 1956, 1966, 1975, 1984, 1994, 2004 et 2014. Ces opérations permettent à chaque fois de répartir la population par sexe, groupe d'âge et état matrimonial aussi bien au niveau national qu'au niveau géographique le plus détaillé (gouvernorats, délégations, communes ou secteurs) mais les résultats ne sont que très rarement disponibles (publiés ou encore tabulables aujourd'hui) à un niveau plus fin que le gouvernorat. Depuis 1975, I'INS produit des estimations annuelles de population par sexe, âge et gouvernorats, auxquelles nous avons pu accéder. Mais ces estimations ne distinguent pas l'état matrimonial.

\section{Qualité des données}

Jusqu'en 2015, l'INS estimait que l'enregistrement des naissances était quasi exhautif depuis 1975 et qu'il n'était plus nécessaire de corriger les données depuis cette date. Comme mentionné plus haut, il semble que I'INS soit un peu revenu sur cette certitude l'an passé en redressant légèrement les résultats des années 2004 à 2014 sans toutefois en préciser ni les raisons ni les moyens (INS, 20I5). En l'absence d'indication sur une détérioration du système d'enregistrement depuis 2004, si une correction est nécessaire, il faudrait qu'elle porte sur toutes les années antérieures. Faute d'information suffisante sur la correction faite par l'INS, il nous semble préférable, dans le cadre de cette étude qui porte sur plusieurs décennies de conserver les séries homogènes dont nous disposons, surtout que la correction en question est assez faible. Nous conservons donc l'hypothèse ancienne de l'INS d'un enregistrement quasi complet depuis 1975, sachant que l'hypothèse de sous-enregistrement était de l'ordre de $5 \%$ pour les années 1965 à 1975 et de 10 $\%$ pour les années 1956-1965 et que les données officielles sont corrigées en conséquence.

La qualité des données ne tient pas seulement au taux de sous-enregistrement, elle touche aussi la précision des informations recueillies, en particulier la déclaration des âges. Il semble qu'en Tunisie les âges déclarés ne soient pas trop loin de la vérité en ce qui concenre l'âge des mères ou celui des jeunes mariées, les unes comme les autres étant nées à une époque où l'état civil était déjà proche de l'exhaustivité. En revanche, il y a un problème non négligeable d'âges non déclarés, dont l'ampleur varie beaucoup avec le gouvernorat. Depuis 2005, la proportion des naissances pour lesquelles l'âge de la mère n'a pas été déclaré a brusquement augmenté dans le Grand Tunis (la capitale et les gouvernorats limitrophes), à Jendouba dans le nord-ouest et à Kasserine dans le centre-ouest. À titre d'exemple en 2010 , alors qu'au niveau national l'âge de la mère est inconnu pour $17 \%$ des naissances déclarées, cette part s'élève à $53 \%$ dans le District de Tunis $(60 \%$ dans le gouvernorat de Tunis, $72 \%$ à l'Ariana, $21 \%$ à Ben Arous, $49 \%$ à Manouba), à 7 I \% dans le gouvernorat de Jendouba et à $42 \%$ dans celui de Kasserine. Cette proportion a également augmenté en 201 I dans le gouvernorat de Tataouine au sud ( $27 \%$ en 201 I et $65 \%$ en 2012 , contre moins de 3 $\%$ auparavant (INS, 20I3). II a fallu redistribuer les naissances d'âge de la mère non déclaré proportionnellement aux âges déclarés. II n'est pas impossible que, dans certains cas, cela fausse un peu les résultats, ce dont il faudra tenir compte dans l'interprétation.

II faut aussi préciser que la question de la domiciliation des naissances, cruciale pour le calcul des taux par gouvernorat, a posé un problème particulier, notamment dans la région de Tunis. Heureusement, I'INS réalise des enquêtes annuelles pour rectifier les résultats sur ce point. Cette rectification n'a cependant pas été faite pour le gouvernorat de la Manouba, dont il ne faudra pas prendre pour acquis les résultats dans l'interprétation.

\section{Le problème des changement territoriaux}

Au lendemain de l'Indépendance, le décret du 21 juin 1956 remplace l'administration territoriale beylicale assez complexe par une organisation plus moderne divisant la Tunisie en 14 gouvernorats ${ }^{i v}$. Ce nombre a http://aps.journals.ac.za 
African Population Studies Vol 30, No 2(Supp.), 2016

été ramené à 13 en 1958 avec la suppression du gouvernorat de

Tozeur dont le territoire est alors rattaché à celui de Gafsa et pour partie à celui de Gabes (figure la). Au milieu des années 1970, il passe à $18^{v}$ (figure Ib), puis à 23 au début des années 1980 avec la création de cinq nouveau gouvernorats ${ }^{\mathrm{vi}}$ (figure Ic) et finalement à 24 avec la création en 2000 du gouvernorat de La Manouba constitué de la partie ouest de celui de l'Ariana (figure Id).

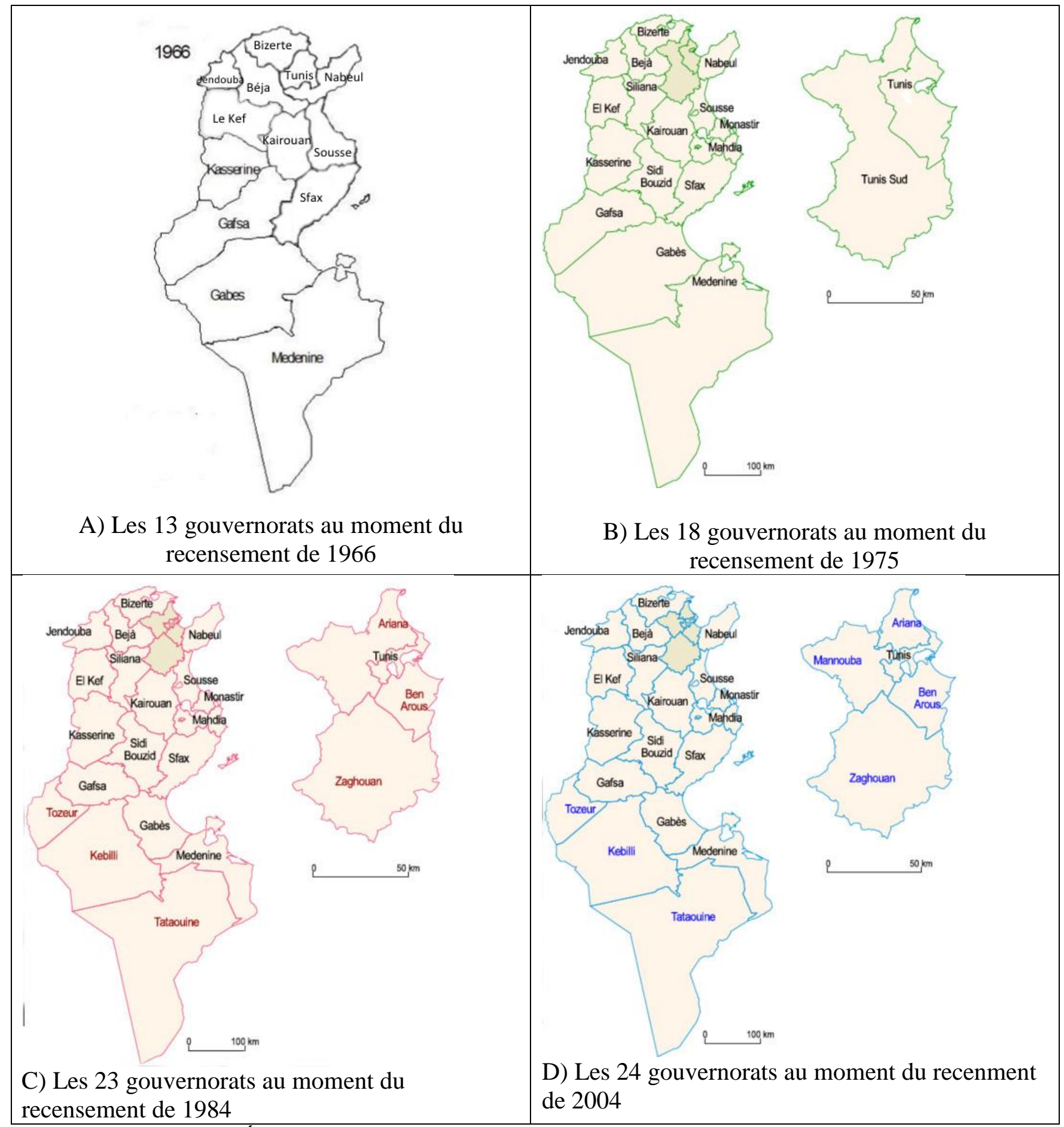

Figure I. Évolution du découpage territorial de la Tunisie d'un recensement à l'autre Sources : carte A : INS, 1973 ; cartes B, C, D : Ben Rabah 201 I

Les modifications opérées au début des années 1970 sont les plus complexes car plusieurs nouveaux http://aps.journals.ac.za gouvernorats sont créés à partir de fractions de territoires de plusieurs anciens gouvernorats. Au 
contraire, les changements suivants ont presque tous été opérés par simple fractionnement d'anciens gouvernorats ${ }^{\mathrm{vii}}$, ce qui facilite la comparaison des résultats (voir les 4 cartes de la figure I). Comme, de toute façon nous n'avons de données sur la fécondité par gouvernorat que depuis le début des années 1970, nous avons décidé de prendre comme point de départ le recensement de 1975 pour lequel les changements de découpage administratif les plus gênants étaient déjà en vigueur.

\section{Indicateurs et mesures}

En dépit de toutes les limites citées ci-dessus, il est possible de calculer l'indicateur de base, le taux de fécondité totale, permettant de suivre l'évolution de la fécondité par gouvernorat depuis 1975 à partir des données annuelles de l'état civil (à l'exception des quelques années manquantes), dans le cadre du découpage géographique du moment.

Pour la nuptialité, ne disposant de données d'état civil que pour les années récentes, nous avons tenté d'approcher l'évolution historique de la géographie de l'âge moyen au premier mariage des femmes en estimant ce dernier par la méthode de Hajnal (1953) sur la base des proportions de célibataires observées aux recensements de 1975, 1984, 1994, 2004 et 2014. Cette méthode pose cependant une difficulté d'interprétation. Elle consiste en effet à utiliser les proportions de célibataires à un âge donné comme les « célibataires subsistants " d'une table de nuptialité : dès lors les différences observées entre deux anniversaires successifs estiment les mariages de la table et l'âge moyen de ces derniers donne l'âge moyen au premier mariage. La méthode combine ainsi les âges aux mariages d'une trentaine de générations qui se sont mariées à des époques différentes et, si comme c'est effectivement le cas pour la Tunisie, les comportements matrimoniaux ont fortement évolué, le résultat obtenu reflète la réalité moyenne d'une période passée dont on ne connait pas aisément les frontières. Nous nous sommes donc efforcés de compléter ces résultats par le calcul de tables de nuptialité reposant sur les mariages enregistrés à l'état civil permettant de suivre précisément l'évolution annuelle de l'âge au mariage. Ces données ne sont que très rarement publiées. Nous avons cependant pu obtenir des tableaux de mariages selon l'âge de l'épouse et son état matrimonial antérieur pour les années 1995 à 20I I au niveau national.

L'évolution de la fécondité des gouvernorats reproduit-elle celle du pays ou bien la géographie de la fécondité a-t-elle changé ?

La figure 2 illustre la baisse très rapide de la fécondité observée au niveau national de 1965 à 1999 évoquée en introduction ainsi que la stabilisation qui a suivi et, finalement, la hausse sensible observée ces toutes dernières années. Cette figure rassemble toutes les estimations disponibles du taux de fécondité totale (TFT), quelle que soit la source : état civil ou enquêtes démographiques. II y a une parfaite concordance entre les résultats estimés à partir de l'état civil (corrigé du sous-enregistrement) et ceux des enquêtes, à la seule exception des ré-estimations récentes de l'INS pour les toutes dernières années.

Au niveau des gouvernorats, compte tenu des données disponible, l'analyse ne peut commencer qu'en 1975 et s'arrête en 2012. D'une façon générale, on retrouve assez bien les trois temps de l'évolution de la fécondité observés au niveau national, même si c'est un peu moins évident pour les années récentes. Toutefois, d'importantes différences de rythme et de calendrier apparaissent.

En 1975, l'écart entre les deux gouvernorats extrêmes était de 4,2 enfants par femme (de 4,2 à Tunis à 8,4 à Sidi Bouzid). En 1999 l'écart maximum n'est plus que de I,4 enfant par femme (entre 1,6 à Tunis et 3,0 à Kasserine) et Sidi Bouzid n'a plus que 0,9 enfants de différence avec Tunis. Dans la période récente, le gouvernorat de Tunis est le seul qui présente une remontée lente mais continue depuis 1999 (passant de 1,6 enfant par femme en 1999 à 2, I en 20I2). Dans quelques autres gouvernorats on observe aussi une remontée, mais beaucoup plus tardive, le plus souvent à partir de 2010 (Bizerte, Sousse, Monastir ou même Kebili). Dans d'autres gouvernorats ce ne sont que fluctuations incertaines (Zaghouan) ou stagnation (Béja, Jendouba, Le Kef). 
TFT (enfants par femme)

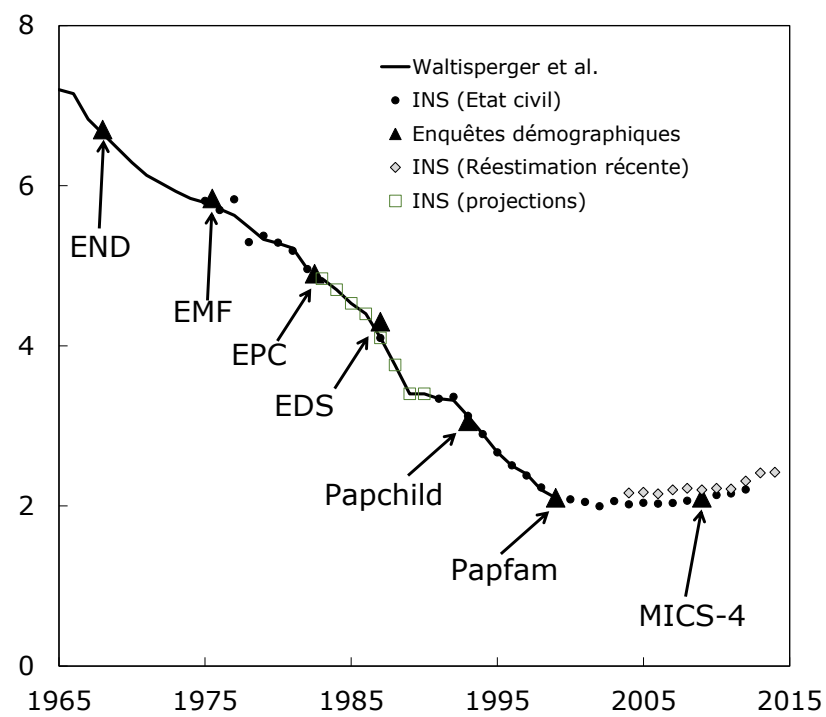

Figure 2 Évolution du taux de fécondité totale au niveau national (1965-2014)

Sources:

- 1966-1995 (serie trait plein): Walperger et al. (200I)

- 1975-20I2 (serie ronds pleins) : état civil (serie intermittante), (INS, divers annuaires statistiques)

- 1983-1990 (serie carrés vides) : Estimations pour les projections de population (INS, 2002),

- 2004-2014 (serie losange gris pleins): Réstimation, (INS, 2015)

- Ponctuellement (serie triangles pleins) Enquêtes spécifiques : Enquête nationale demographque (END) (INS,

1974), Enquête sur la prévalence contracepive (EPC) (ONFP, 1985) Enquête mondiale fécondité (EMF)

(ONFP, 1982), Enquête démographique et de santé (EDS) (Aloui et al.), Enquête tunisienne sur la santé de la mère et de l'enfant (projet Papchild) (ONFP, 1997); Enquête tunisienne sur la santé de la famille (projet Papfam)

(ONFP, 2002) ; Suivi de la situation des enfants et des femmes (projet MICS4) (MDCl et al., 20/3)

En dépit de tendances générales comparables, on note donc une évolution de la structure géographique de la fécondité, peut être encore plus marquée dans la période récente, sans doute en partie parce qu'elle est une période de stabilisation.

Les cartes de la figure 3 illustrent les transformations successives de la carte de la fécondité tunisienne d'une date à l'autre, de 1975 à 2012. Six cartes sont présentées, pour les années 1975, 1984, 1989, 1999, 2009 et 2012. L'année 1999 est celle du changement de tendance entre la période de baisse très rapide de la fécondité et la période de sa stabilisation, telles que la figure 2 permet de les identifier au niveau national. Les deux premières années sont celles des recensements de 1975 et 1984. Nous y avons ajouté 1989 pour éviter une trop longue distance entre 1984 et $1999 ; 2009$ permet d'illustrer la fin de la période de stricte stagnation et
2012 (dernière année disponible) de voir si la légère hausse récente produit un effet sur la géographie. Les représentations sont faites à échelle de valeurs constante (allant de I enfant pas femme, valeur minimum observée en 2012 , à 7,45 , valeur maximale observée en 1975) de façon à ce que l'on suive correctement les changements du niveau de la fécondité en même temps que ses variations géographiques. II est vrai que cela applatit sensiblement le relief caractérisant chaque époque mais cela évite les fausses interprétations. 


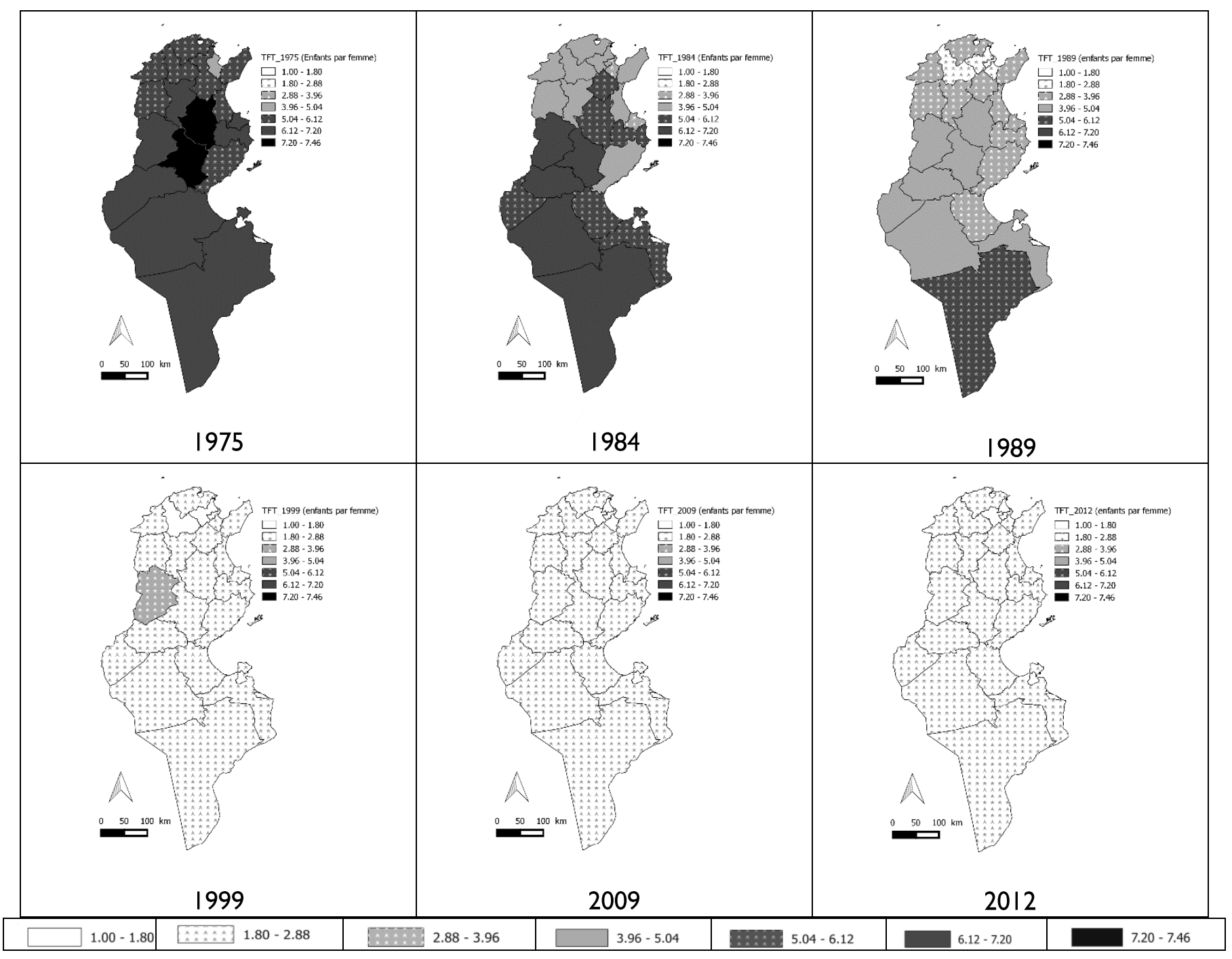

Figure 3. Évolution de la carte du taux de fécondité totale par gouvernorat de 1975 à 2012

Afin de bien suivre les changements de niveau, les cartes sont tracées sur la base d'une échelle de valeur constante. De 1975 à 1999, la carte passe de trames très foncées à des trames très claires en raison de la grande baisse de la fécondité.

De 1999 à 2012, elle reste constamment claire, du fait de la stabilisation.

De manière caractéristique, de la première carte (1975) à la dernière $(20 / 2)$ on passe d'une carte dominée par la haute fécondité (avec des nuances entre des gouvernorats à très haute fécondité et d'autres à fécondité un peu moins forte), à une carte dominée par des fécondité toutes très proches du niveau de remplacement (presque sans nuances). Mais, de l'une à l'autre, on est passé par des phases d'évolution très différentes. Dans une première phase, de 1975 à 1989, on assistante à une forte divergence. Puis la divergence a cédé la place à une convergence extrêmement forte et rapide qui en dix ans, de 1989 à 1999, efface presque tout relief. Et, finalement la période de stabilisation et même celle de légère hausse récente (au niveau national) vont de pair avec le maintien apparent de cet effacement du relief géographique de la fécondité.

\section{Divergence}

En 1975, alors que la fécondité tunisienne était encore très élevée (près de six enfants par femme en moyenne au niveau national), la moitié des gouvernorats (9 sur 18) avaient une fécondité supérieure à la moyenne, parfois très forte comme à Kairouan et Sidi Bouzid avec plus de 7 enfants par femme. Ces deux gouvernorats du centre-est étaient entourés par sept autres où la fécondité se situait entre 6,2 et 7,2 enfants par femme, à savoir les trois gouvernorats du sud (Gafsa, Gabes et Medenine), deux autres gouvernorats du centre-est (Siliana et Kasserine) et deux du Sahel (Monastir et Mahdia). La fécondité moins forte caractérisait, au contraire, le nord et l'ouest de la Tunisie. Le minimum était atteint à Tunis (4,2 enfants par femme) les huit autres gouvernorats se situant entre 5 et 6 enfants par femme : Bizerte, Béjà, Jendouba, Le Kef au nord et Zaghouan, Nabeul, Sousse et Sfax à l'est.

En 1984 puis en 1989, ce contraste nordouest/sud-est parait s'être accentué du fait que la 
grande baisse de la fécondité tunisienne a commencé par toucher les gouvernorats du nord, de la région de Tunis et du Sahel. En 1984, presque tous les gouvernorats du sud et du centre-est sont encore des zones à haute fécondité, tandis que ceux du nord et de la côte méditerranéenne ont déjà fortement réduit leur fécondité (entre 4 et 5 enfants par femme), les gouvernorats de Tunis et de Monastir étant même descendus à moins de 4. En 1989, il ne reste plus au sud qu'un gouvernorat à très forte fécondité mais le contraste nord-est/sud-ouest paraît nénmoins renforcé, car tout le nord et l'est sont à moins de 4 enfants par femme et 4 gouvernorats du nord sont à moins de 3 (Tunis, Beja, Ariana, Ben Arous). En 1989, la carte de la fécondité tunisienne identifie clairement quatre ensembles bien compacts opposant la région de Tunis à fécondité très basse au grand sud à fécondité encore très élevée, en passant par un ensemble, nord-est, à fécondité assez basse et un autre, centre-ouest, à fécondité encore assez élevée. C'est en effet, dans cette première phase, que les régions du nord et de l'est de la Tunisie ont connu les baisses de fécondité les plus fortes, là même où la fécondité était déjà au départ moins élevée (tableau I).

Tableau I. Taux de fécondité totale (TFT) de l'année et évolution relative durant la période, par gouvernorat.

\begin{tabular}{|c|c|c|c|c|c|c|c|c|c|c|c|}
\hline \multirow{2}{*}{ Gouvernorat } & \multicolumn{6}{|c|}{ TFT } & \multicolumn{5}{|c|}{ Évolution relative du TFT par période(*) } \\
\hline & 1975 & 1984 & 1989 & 1999 & 2009 & 2012 & 1975-84 & 1984-89 & 1989-99 & 1999-09 & $2009-12$ \\
\hline Tunis & 4,2 & 3,5 & 2,4 & 1,6 & 1,8 & 2,1 & $-14,8$ & $-30,9$ & $-35,7$ & 16,1 & 15,6 \\
\hline Ben Arous & & 3,8 & 2,7 & 2,1 & 1,5 & 1,9 & & $-30,3$ & $-22,3$ & $-25,2$ & 25,3 \\
\hline Zaghouan & 5,5 & 5,8 & 3,2 & 2,3 & 2,2 & 2,3 & $-18,1$ & $-45,4$ & $-26,3$ & $-7,3$ & 6,0 \\
\hline Ariana & & 4,1 & 2,8 & 1,7 & 1,5 & 1,8 & & $-30,6$ & $-39,4$ & $-9,6$ & 19,3 \\
\hline Manouba & & & & & 1,6 & 1,0 & & & & & $-38,7$ \\
\hline Nabeul & 5,8 & 4,4 & 3,1 & 2,0 & 2,2 & 2,3 & $-24,0$ & $-30,1$ & $-34,3$ & 7,9 & 4,1 \\
\hline Bizerte & 5,1 & 4,0 & 3,1 & 1,9 & 1,9 & 2,3 & $-20,5$ & $-22,3$ & $-38,3$ & $-2,1$ & 22,2 \\
\hline Beja & 5,4 & 4,0 & 2,7 & 1,8 & 1,9 & 1,9 & $-26,4$ & $-33,0$ & $-34,7$ & 6,9 & 2,1 \\
\hline Jendouba & 5,3 & 4,6 & 3,4 & 2,2 & 1,9 & 1,9 & $-13,9$ & $-25,3$ & $-34,7$ & $-13,1$ & $-2,1$ \\
\hline Le Kef & 5,2 & 4,3 & 3,1 & 1,9 & 2,0 & 1,9 & $-18,6$ & $-27,0$ & $-37,9$ & 5,2 & $-4,4$ \\
\hline Siliana & 6,3 & 4,6 & 3,2 & 2,3 & 2,1 & 2,3 & $-26,8$ & $-30,5$ & $-30,1$ & $-7,6$ & 12,0 \\
\hline Kasserine & 7,1 & 6,6 & 4,8 & 3,0 & 2,5 & 2,5 & $-6,4$ & $-28,0$ & $-38,0$ & $-13,9$ & $-2,4$ \\
\hline Sidi Bouzid & 7,5 & 6,3 & 4,6 & 2,4 & 2,5 & 2,2 & $-15,6$ & $-27,2$ & $-46,7$ & 0,4 & $-10,2$ \\
\hline Gafsa & 6,9 & 6,2 & 4,3 & 2,1 & 2,2 & 2,3 & $-13,9$ & $-30,8$ & $-50,8$ & 5,7 & 3,2 \\
\hline Tozeur & & 5,3 & 4,0 & 2,2 & 2,4 & 2,4 & & $-24,6$ & $-44,5$ & 9,0 & $-2,1$ \\
\hline Médenine & 6,9 & 5,5 & 4,3 & 2,2 & 2,1 & 2,4 & $-16,4$ & $-22,1$ & $-48,1$ & $-7,6$ & 16,0 \\
\hline Tataouine & & 6,6 & 5,5 & 2,7 & 2,0 & 2,2 & & $-16,9$ & $-51,1$ & $-26,2$ & 9,6 \\
\hline Gabes & 6,8 & 5,7 & 3,9 & 2,2 & 2,1 & 2,3 & $-11,5$ & $-31,6$ & $-44,4$ & $-5,0$ & 12,6 \\
\hline Kebili & & 6,9 & 5,0 & 2,0 & 2,2 & 2,5 & & $-27,8$ & $-59,4$ & 6,4 & 16,7 \\
\hline Sfax & 5,3 & 4,1 & 3,2 & 2,0 & 2,1 & 2,2 & $-23,7$ & $-22,0$ & $-36,7$ & 3,0 & 5,3 \\
\hline Kairouan & 7,2 & 5,7 & 4,1 & 2,5 & 2,4 & 2,3 & $-21,3$ & $-28,5$ & $-39,3$ & $-2,0$ & $-3,3$ \\
\hline Sousse & 5,8 & 4,5 & 3,4 & 2,0 & 2,2 & 2,7 & $-23,4$ & $-23,5$ & $-40,8$ & 9,4 & 21,7 \\
\hline Monastir & 6,2 & 3,9 & 3,7 & 2,4 & 2,3 & 2,8 & $-36,5$ & $-7,1$ & $-34,2$ & $-4,6$ & 22,3 \\
\hline Mahdia & 6,5 & 5,4 & 3,9 & 2,6 & 2,4 & 2,5 & $-16,8$ & $-28,5$ & $-33,2$ & $-8,5$ & 5,0 \\
\hline
\end{tabular}

(*) Pour calculer l'évolution relative du TFT dans le gouvernorat de Tunis entre 1975 et 1984, nous avons calculé un TFT en 1984 à Tunis dans les frontières de 1975 en faisant la moyenne des TFT des gouvernorats comprenant en 1984 une partie du gouvernorat de Tunis de 1975, pondérée par la population de ces parties.

\section{Convergence}

Au contraire, et très rapidement, de 1989 à 1999 , alors que la baisse de la fécondité s'accélérait au niveau national, celle-ci a été la plus forte dans les gouvernorats où elle n'avait pas encore beaucoup baissé.

Ainsi, en 1999, le relief géographique de la fécondité tunisienne s'est érodé, la carte est 
désormais presque uniforme, autour du niveau de remplacement, à deux exceptions près : le gouvernorat de Kasserine, à l'ouest, où la fécondité reste légèrement supérieure à 3 enfants par femme et la région de Tunis (gouvernorats de Tunis, Béja et Ben Arous) où celle-ci est inférieure à I,8 enfant par femme. De 1989 à 1999 les rythmes de baisse se sont inversés entre le nord-est et le sud-ouest. La fécondité a baissé de plus de $50 \%$ à Gafsa, Kebili et Tataouine, contre moins de $30 \%$ à Zaghouan et Ben Arous.

La période de stabilisation de la fécondité au niveau national ne fait que renforcer cette convergence. En 2009, en effet, le contraste est encore plus faible qu'en 1999, n'opposant plus que Tunis et ses voisins à tout le reste du pays. Et la légère remontée de féconté depuis 2009 parait avoir encore accentué cet effacement, Tunis et Ben Arous étant repassés au dessus de 2 enfants par femme, ne laissant plus en deçà que l'Ariana (et Manouba qui en $a$ été détaché en 2000 , mais dont la très basse fécondité affichée est fortement sous-estimée à cause des erreurs de domiciliation).

Disparités géographiques de la nuptialité : l'inversion du relief

Au niveau national, nous disposons de données permettant une mesure directe de l'âge moyen au premier mariage (nombre de premiers mariages déclarés à l'état civil selon l'âge des femmes et l'état matrimonial antérieur, et estimation de la population par âge et sexe selon l'état matrimonialviii) pour chaque année de 1995 à $201 \mathrm{I}$. Nous avons donc pu construire des tables de primo-nuptialité annuelles pour cette courte période qui permettent de suivre l'évolution récente de l'AMPM (ligne noire épaisse de la figure 4). Malgré quelques fluctuations, cette dernière indique clairement que cet indicateur suit une tendance stable.

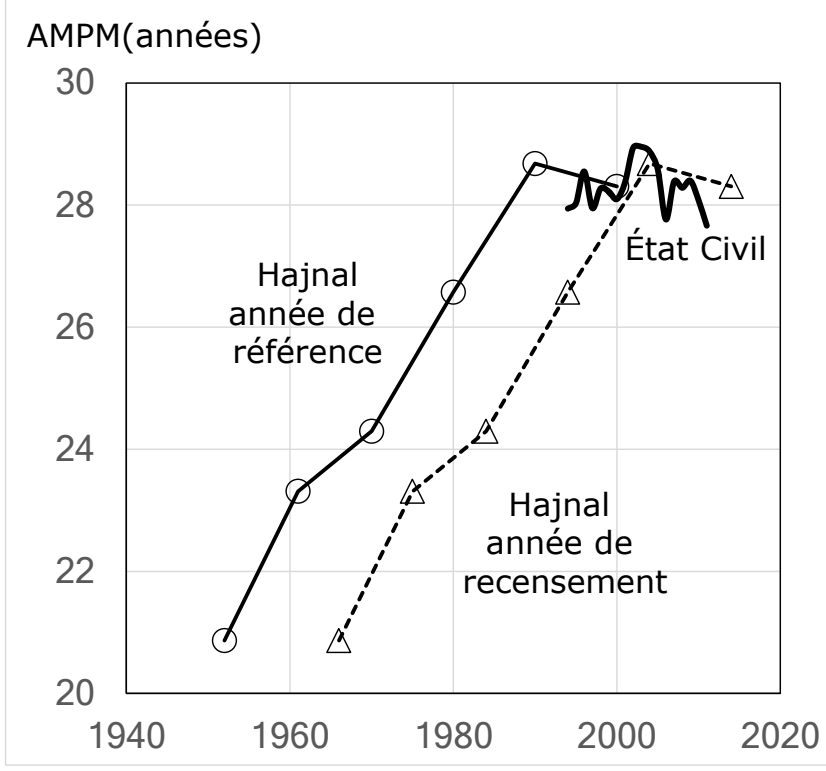

Figure 4. Évolution de l'âge moyen au premier mariage (AMPM) des femmes en Tunisie, depuis 1950 Trait épais : calcul direct à partir des mariages enregistrés à l'état civil. Cerlces : estimations indirectes à partir des proportions de célibataires par âge aux recensementx positionnées à l'année de recensement. Triangles : les mêmes positionnées I 4 ans en arrière (centre de la période de référence de l'estimation). Calculs des auteurs à partir des recensements

Pour remonter plus loin dans le temps, il faut se contenter d'une mesure indirecte, par la méthode de Hajnal. Cela permet d'enrichir la figure de six points correspondant aux recensements de 1966, 1975, 1984, 1994, 2004 et 2014 (triangles sur la figure 4). Les deux derniers points (2004 et 2014) semblent être en parfaite cohérence avec la ligne des mesures directes mais celui de 1994, très inférieur à ce que donne la meusure directe pour l'année suivante $(26,6$ ans au lieu de 28 en 1995), ne l'est pas du tout. En réalité cela n'est pas surprenant car, justement, la 2593 population tunisienne est loin d'avoir été stable (ne serait-ce qu'en raison de la chute de sa fécondité) tandis que, comme l'indiquent les points antérieurs, l'âge moyen au premier mariage a connu une très forte augmentation. Dans ces conditions, il faut absolument, pour retracer l'historique de cette évolution de la nuptialité, tenir compte du décalage dans le temps qui sépare la période de référence de l'âge moyen au mariage mesuré pas la méthode de Hajnal et la date du recensement utilisé à cette fin. Les mariages qui ont fait que la proportion de http://aps.journals.ac.za 
célibataire à un âge donné est moins forte qu'à l'âge précédent n'ont que très rarement eu lieu durant l'année de recensement, mais le plus souvent au cours d'une année antérieure d'autant plus lointaine que l'âge est élevé. II faut donc en tenir compte pour placer correctement dans le temps les âges moyen mesurés par la méthode de Hajnal par rapport à ceux qui ont pu être mesurés directement. Ce que l'on ne peut justement pas faire sur la base de données qui en interdisent la mesure directe.

Nous avons cependant pu avoir accès à partir du recensement de 2014 à une information rarement disponible à partir des recensements : l'âge au premier mariage déclaré des femmes non célibataires recensées. La différence entre cet âge et leur âge au recensement permet de retrouver l'année de leur premier mariage et finalement de calculer l'année moyenne de conclusion des mariages qui ont été pris indirectement en compte par différence entre proportions de célibataires. En 2014, cette année moyenne s'est avérée être de 14 ans antérieure à l'année du recensement. Rien ne prouve que cet écart soit constant dans le temps. On peut néanmoins, faute de mieux, faire cette hypothèse et déplacer de 14 ans en arrière le positionnement des AMPM résultant des 6 recensements (cercles reliés par une ligne continue de la figure 4). Dès lors, les points correspondants aux recensements apparaissent en bonne cohérence avec la ligne noire des AMPM calculés directement. II apparaît que la stabilisation de l'AMPM date de la deuxième moitié des années 1980. Et c'est du début des années 1950 au tournant des années 1990 que l'AMPM s'est élevé de 20,8 ans (vers 1956) à 28,7 (vers 1990) avant de se stabiliser. On constate au passage que cette montée de l'AMPM a démarré au moins une dizaine d'années avant la promulgation de la loi de 1964 imposant un âge minimum de 17 ans au mariage des jeunes filles. En dépit de l'effet secondaire qu'elle a provoqué (voir ci-dessus), cette loi n'a probablement été rendue acceptable que parce que cette hausse de l'âge moyen au mariage avait déjà été amorcée par l'évolution de la société tunsienne.

On constate aussi que cette hausse remarquable de l'AMPM de la fin des années 1950 à la fin des années 1980 est presque parfaitement symétrique, à une dizaine d'années de distance, à la chute tout aussi remarquable de la fécondité des années 1960 aux années 1990, cette dernière étant, comme on le sait, fortement liée à la première.

Qu'en est-il au niveau des variations géographiques ? Retrouve-t-on en matière d'AMPM une structure géographique symétrique à celle de la fécondité et son évolution explique-t-elle celle de la fécondité ?

On ne peut estimer l'AMPM au niveau des gouvernorats qu'indirectement, selon la méthode de Hajnal (tableau 2). Les évolutions sont, en gros, parallèles à celles observée au niveau national. On note cependant, comme pour la fécondité, quelques différences. Durant la période d'élévation de l'AMPM, le rythme d'augmentation varie sensiblement. II est, par exemple plus récent mais aussi plus rapide dans les gouvernorats de Kebili et Tataouine $^{\mathrm{ix}}$ au sud qui contrastent avec la montée beaucoup plus lente observée à Tunis. Plus nettement encore, la stabilisation observée dans la période 1990-2000 s'avère être la résultante d'une divergence entre des gouvernorats où l'AMPM continue à augmenter comme à Tataouine et d'autres où il diminue profondément comme à Tunis.

Tableau 2. Âge moyen au premier mariage des femmes par gouvernorat, estimé par la méthode de Hajnal à partir des proportions de célibataires observées aux recensements

\begin{tabular}{|c|c|c|c|c|c|}
\hline Gouvernorats & $\begin{array}{l}C=1975 \\
R=1961\end{array}$ & $\begin{array}{l}C=1984 \\
R=1970\end{array}$ & $\begin{array}{l}C=1994 \\
R=1980\end{array}$ & $\begin{array}{l}C=2004 \\
R=1990\end{array}$ & $\begin{array}{l}C=2014 \\
R=2000\end{array}$ \\
\hline Tunis & 24,3 & 25,8 & 28,0 & 29,6 & 28,6 \\
\hline Ben Arous & & 25,0 & 27,1 & 28,4 & 28,2 \\
\hline Zaghouan & 23,6 & 24,5 & 26,9 & 28,6 & 27,5 \\
\hline Ariana & & 24,3 & 26,7 & 28,1 & 28,0 \\
\hline Manouba & & & & 29,3 & 28,4 \\
\hline Nabeul & 23,7 & 24,7 & 26,2 & 27,7 & 27,2 \\
\hline Bizerte & 23,9 & 24,3 & 26,3 & 28,1 & 28,0 \\
\hline Beja & 23,9 & 24,8 & 27,3 & 29,0 & 28,2 \\
\hline Jendouba & 23,9 & 24,8 & 27,0 & 28,8 & 28,7 \\
\hline Le Kef & 23,5 & 24,9 & 27,5 & 29,3 & 28,5 \\
\hline Siliana & 22,9 & 24,1 & 27,0 & 29,2 & 27,7 \\
\hline Kasserine & 21,5 & 22,8 & 25,7 & 28,6 & 29,3 \\
\hline Sidi Bouzid & 21,7 & 23,5 & 27,0 & 30,1 & 29,2 \\
\hline Gafsa & 22,4 & 23,9 & 27,1 & 30,1 & 29,3 \\
\hline Tozeur & & 24,6 & 27,0 & 29,5 & 28,9 \\
\hline Médenine & 21,0 & 21,9 & 25,0 & 28,8 & 29,9 \\
\hline
\end{tabular}


Tataouine

Gabes

Kebili

Sfax

Kairouan

Sousse

Monastir

Mahdia

$\mathrm{C}=$ année de recensement source de l'estimation

$\mathrm{R}=$ année moyenne de la période de référence

La mesure de l'AMPM reposant entièrement sur les recensements, nous ne pouvons pas dessiner des cartes aux mêmes dates que pour la fécondité. En particulier nous ne pouvons pas nous référer à 1999 et 2009, années des 2 principaux points de ruptures qui jalonnent l'évolution de cette dernière depuis 1975. Cela n'est pas vraiment gênant puisque l'évolution de l'AMPM est très décalée dans le temps par rapport à celle de la fécondité. Mais nous ne pouvons pas non plus, comme cela serait logique, remonter le temps en deçà de 1975 sans nous heurter aux changements trop importants de découpage géographique opérés au début des années 1970. Nous allons donc nous contenter de représenter la géographie de la nuptialité aux cinq derniers recensements : 1975, 1984, 1994, 2004 et 20I4. Comme pour la fécondité, pour chaque figure, l'échelle des valeurs est la même pour les cinq cartes. Bien sûr, comme on vient de le dire, les mesure faites à partir des recensements valent pour des périodes de référence dont la moyenne se situe 14 ans avant le recensement, c'est à dire en 1961, 1970, 1980, 1990 et 2000.

Les cinq cartes de la figure 5 illustrent la transformation de la structure géographique de l'AMPM. Dans l'ensemble, on passe d'une situtation où, partout, le mariage est précoce ou très précoce à une nouvelle carte enièrement marquée par le mariage tardif, à mesure que l'AMPM national augmente. Cependant, en 40 ans la géographie de l'âge au premier mariage a radicalement changé. Vers I96I, si les femmes se mariaient jeunes partout, on observait tout de même un net contraste entre, d'une part, le sud et le centre-Ouest, où les femmes se mariaient particulièrement tôt, et d'autre part, le reste du pays où l'AMPM se situait entre 22 et 24 ans et même un peu plus à Tunis et Monastir. Vers 1970 le relief s'accentue avec un dégradé en quatre tranches d'âges allant du sud vers le centre-ouest, puis le nord et l'est et finalement Tunis. Vers 1980, le profil reste à peu près le même mais avec des AMPM partout nettement plus élevés. Vers 1990, l'AMPM a encore beaucoup augmenté mais davantage au sud qu'au nord et le profil géographique s'est brouillé. Vers 2000 , enfin, on retrouve un dégradé très net distinguant sud, centre-ouest et nord-et-est, mais complètement inversé car cette fois c'est au sud que les AMPM sont les plus élevé et au nord et à l'est qu'ils sont les moins élevés.

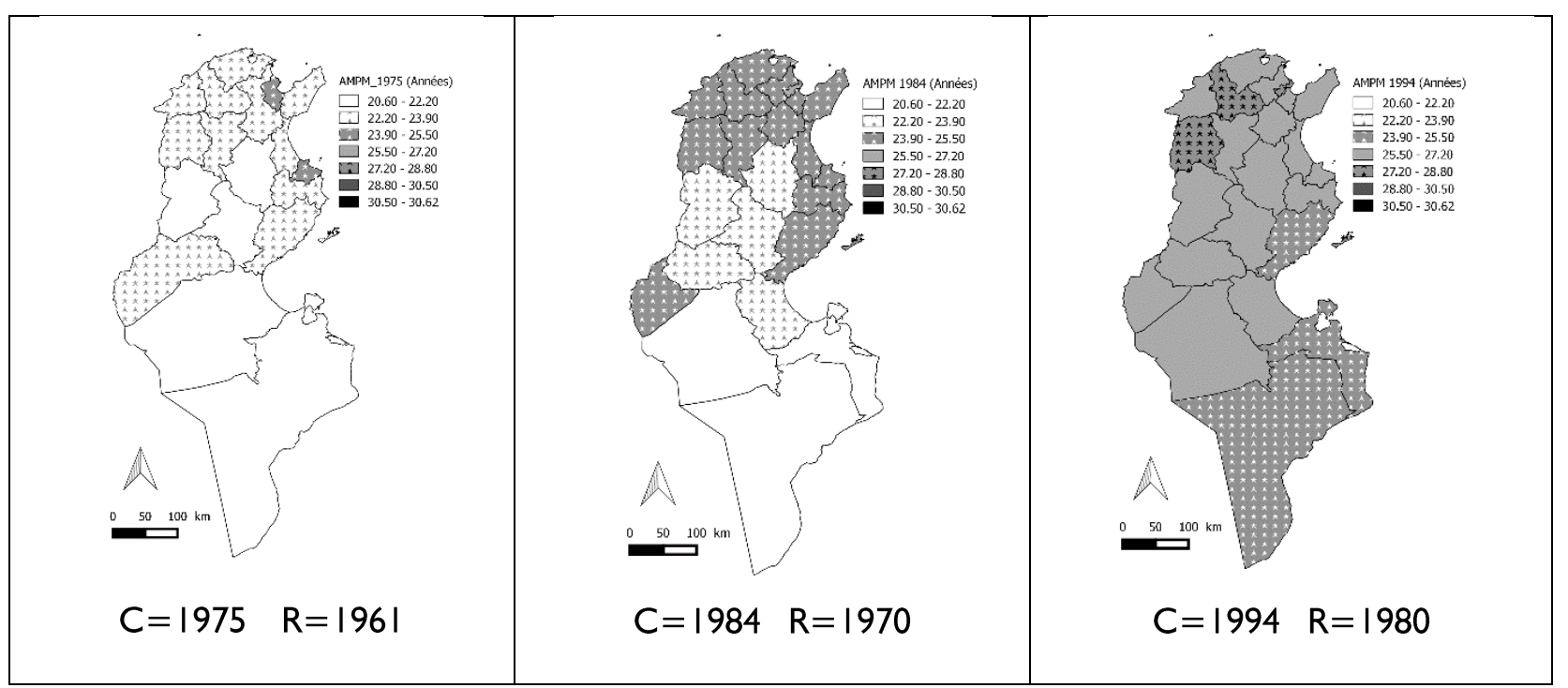




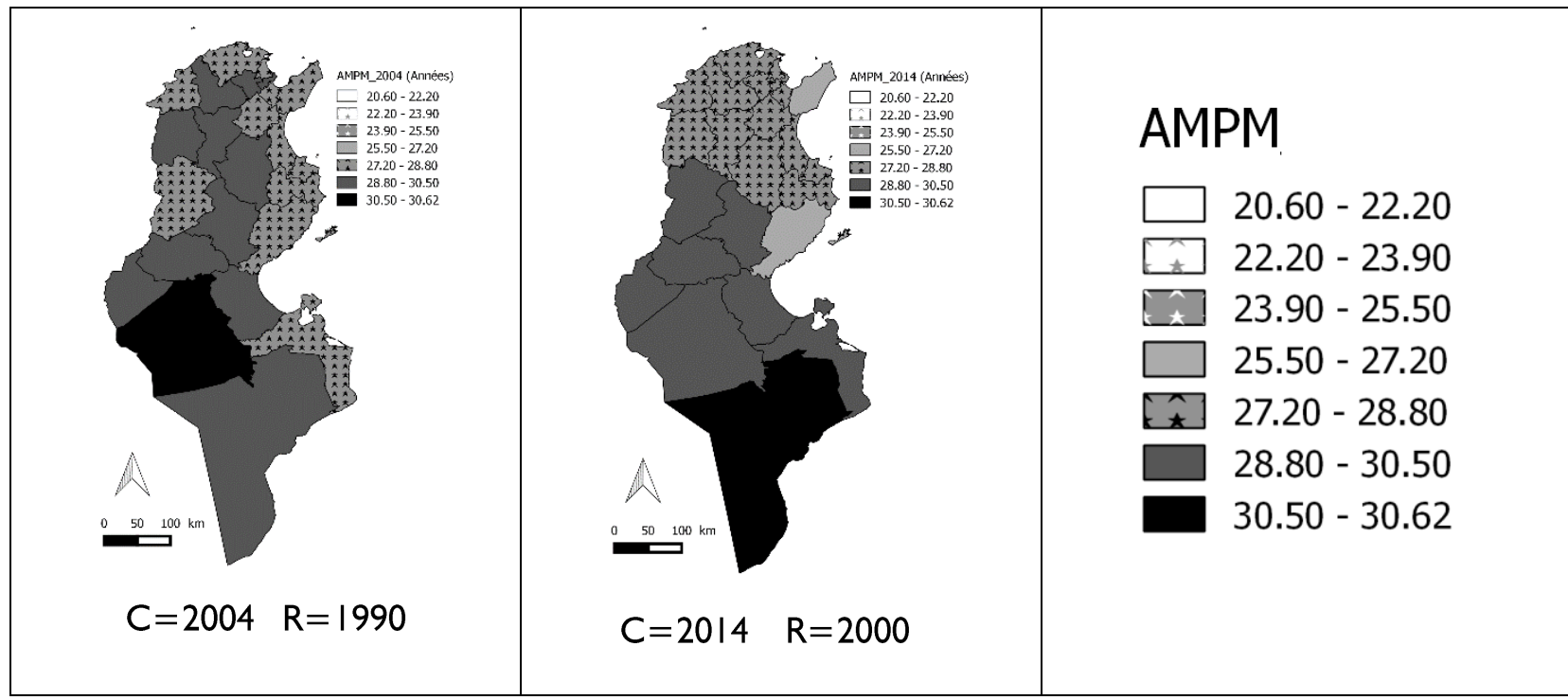

Figure 5. Évolution de la carte des âges moyens au premier mariage (AMPM) par gouvernorat de 1975 à 2014 , calculés par la méthode de Hajnal

Afin de bien suivre les changements de niveau, les cartes sont tracées sur la base d'une échelle de valeur constante. Les AMPM passent de trames très claires aux trames très foncées à mesure qu'ils augmentent.

$C=$ année de recensement source de l'estimation

$R=$ année moyenne de la période de référence

Du côté de la fécondité, la figure 4 avait aussi mis en évidence une inversion du profil géographique du TFT, cependant l'inversion était moins violente, la carte la plus récente résultant beaucoup plus d'un arasement du profil que de son inversion : seuls les gouvernaorats de l'Ariana et Manouba au nord et de Sousse et Monastir à l'est s'opposaient à tout le reste du pays complètement monochrome.

Cela n'empêche évidemment pas de se demander s'il existe une relation entre les variations géographiques de la fécondité et celles de la nuptialité, même si l'on peut s'attendre à ce qu'elles soient assez faibles.

Y a-t-il un lien entre les disparités géographiques de la fécondité (TFT) et celles de la nuptialité (AMPM) ?

La question n'est pas ici de savoir si, au niveau régional, dans chaque gouvernorat, la baisse de la fécondité est liée à l'élévation de l'âge au mariage. La figure 5 montre assez clairement que partout l'âge au mariage a fortement augmenté tandis que partout la fécondité a rapidement diminué (figure 3 ) pour comprendre que, gouvernorat par gouvernorat, on retrouverait la même symétrie qu'au niveau national entre l'élévation de l'âge au mariage (figure 4) et la baisse de la fécondité (figure 2). Certes la différence de nature entre des TFT mesurés directement et des AMPM mesurés indirectement brouille assez fortement la confrontation des calendriers respectifs de ces deux phénomènes. En effet, le décalage de 14 ans utilisé pour les AMPM, très grossier, force à dater de la même année des mariages qui se sont échelonnés sur des périodes plus ou moins longues pouvant dépasser la trentaine d'années. Mais de toute évidence, l'élévation de l'âge au mariage a précédé la baisse de la fécondité. Et une étude cas par cas montrerait que partout, comme au niveau national, la montée de l'âge au mariage a été un facteur décisif de la baisse de la fécondité, avant même que la diffusion de la contraception ait pu y contribuer et prendre le relais.

II s'agit ici de voir si, plus précisément, il existe aussi un lien entre les variations de la structure géographique de la fécondité et celles de la nuptialité. Pour ce faire, il faut évidemment distinguer deux grandes périodes : celle où la fécondité baissait et celle où elle s'est stabilisée. Nous nous limiterons sur ce point à la période 1975-1999, durant laquelle la fécondité a baissé au niveau national, mais aussi presque partout dans le pays.

Bien qu'il soit difficile de faire précisément le partage, il est préférable de distinguer deux périodes car il semble que la fécondité ait continué à baisser alors que l'âge au mariage avait déjà cessé d'augmenter. Les relations entre les deux indicateurs risquent donc d'être différentes, si toutefois on peut les mesurer correctement.

Compte tenu des données disponibles, on peut d'abord se référer à la période 1980-1990 où l'âge au mariage augmente, puis à la période 1990-2000 où il se stabilise. Compte tenu de l'incertitude sur le décalage de 14 ans pris en compte pour les AMPM calculés indirectement, surtout pour les périodes les plus anciennes, on peut comparer l'evolution de l'AMPM durant ces deux périodes à celle du TFT 
durant les périodes 1975-1989 et 1989-1999 respecti-vement.

La figure 6 compare donc deux périodes de baisse de la fécondité à deux périodes à peu près concomittantes d'évolution contrastée de la nuptialité : 1975-89 pour la fécondité et 1980-90 pour l'âge au mariage (en cours d'augmentation), puis 1989-99 pour la fécondité et 1990-2000 pour l'âge au mariage (en cours de stabilistaion). Les correlations sont faibles dans les deux cas, mais de sens opposés.

\section{Lorsque l'AMPM augmente et que le TFT diminue au niveau national}

Dans ce premier cas, elle est positive avec un coefficient de corrélation faible $R=0,27$ : plus l'âge au mariage augmente, moins la fécondité diminue (figure $6 \mathrm{~A}$ ). Cela veut dire que dans les gouvernorats où la fécondité décroit le plus vite, l'augmentation de l'âge au mariage est la moins rapide. La relation semble donc contredire l'influence négative sur la fécondité de l'élévation de l'âge au mariage. Certes, la correlation est faible mais il doit aussi y avoir dans les variantes entre gouvernorats des éléments qui contrecarrent en partie la relation principale. De fait, dans la période 1970-80 le relief géographique de la

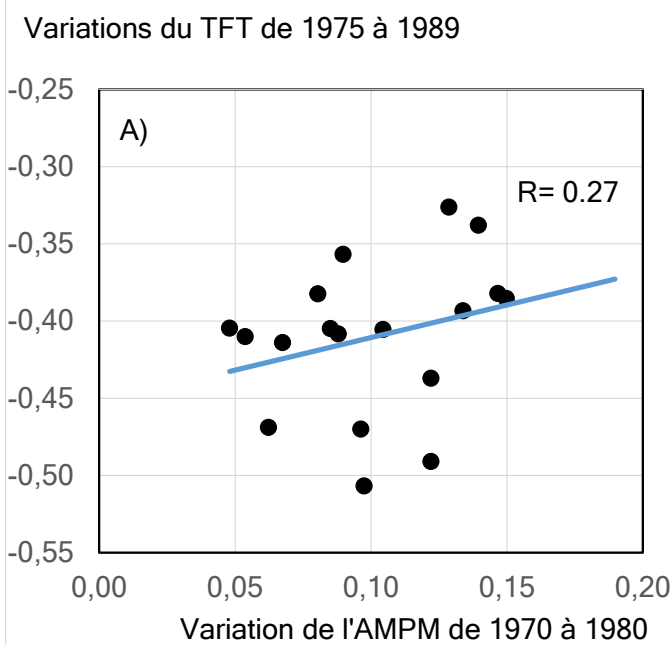

nuptialité a fortement changé, avec une montée rapide de l'âge au mariage dans les gouvernorats du sud, alors que cette montée se ralentissait au nord et à l'est où la hausse de l'âge au mariage avait commncé beaucoup plus tôt. En revanche, côté fécondité, le rythme de baisse n'était guère différent au sud de ce qu'il était partout ailleurs dans le pays sans doute en grande partie parce que la contraception s'est diffusée de façon plus homogène à travers le pays, grâce au programme national de planning familial qui battait son plein au tournant des années 1980. En réalité, on peut aussi penser que si la correlétion est faible, c'est que les périodes ne sont pas très bien choisies. II faudrait comparer les variations de fécondité à celles de la nuptialité dans des périodes plus courtes et convenablement décalées dans le temps. Ce que nous ne pouvons faire compte tenu des dates de recensements (espacées de 10 ans) à partir desquels les âges au mariage sont estimés.

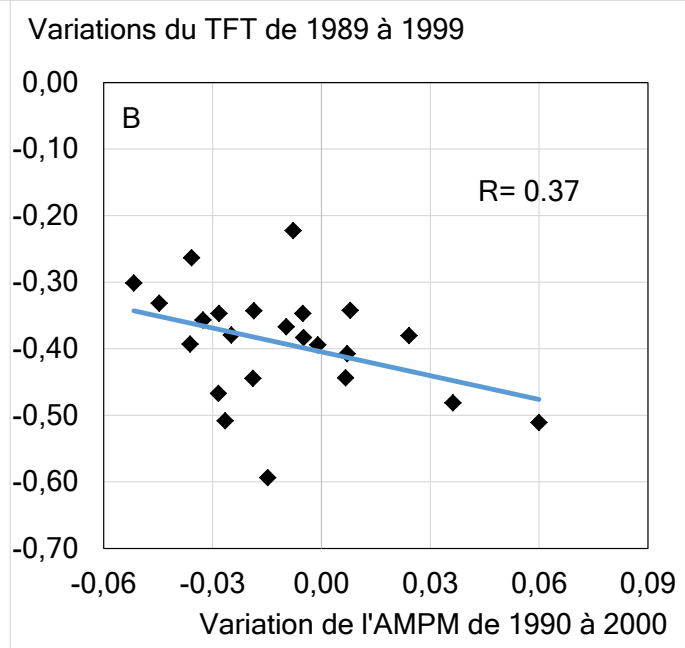

Figure 6. Corrélations entre évolution du TFT et celle de l'AMPM au cours de deux périodes de baisse de la fécondité

A) Quand l'âge au mariage augmente

B) Quand l'âge au mariage n'augmente plus

Lorsque l'AMPM se stabilise et que le TFT continue à diminuer au niveau national

Dans ce second cas, la correlation est un peu moins faible, mais elle est cette fois négative avec un $\mathrm{R}=0,37$ : plus l'âge au mariage augmente, plus la fécondité diminue (figure $6 \mathrm{~B}$ ). Cette fois-ci la relation est dans le sens attendu, mais elle n'est pas très forte.

De fait, c'est la période où c'est dans le sud que la baisse de la fécondité est la plus forte alors qu'elle s'est, au contraire, ralentie dans la région de Tunis.
C'est aussi la période où le sud rattrape le nord en matière d'élévation de l'âge au mariage. Cela efface en grande partie les facteurs qui, dans la période précédente, contrariaient l'effet négatif de la hausse de l'âge au mariage sur la fécondité, mais pas assez pour donner une correlation forte.

\section{Conclusion}

La fécondité tunisienne a commencé à baisser dans la seconde moitié des années 1960 d'abord sous l'effet 
d'une forte augmentation de l'âge moyen au premier mariage des femmes, avant même que la diffusion de la contraception ne vienne renforcer ce mouvement. De 7,5 en 1965, le nombre d'enfants par femme est tombé à 2,I en 1999. Depuis, il s'est stabilisé avant de remonter légèrement après 2009, pour atteindre 2,4 en 2014 , selon les dernières estimations de l'Institut national de la statistique tunisien. La cartographie des niveaux de fecondité par gouvernorat et son évolution depuis 1975 a montré que la baisse de la fécondité tunisienne et sa stabilisation récente se sont accom-pagnées de forts changements dans sa structure géographique. Durant la période de baisse, les niveaux de fécondité ont d'abord divergé, accuentuant le relief geographique de la fécondité, puis ont conmmencé à converger, convergence qui s'est accentuée avec la stabilisation et que la légère remontée récente n'a pas remise en cause. Des mouvements de divergence-convergence ont également eu lieu pour l'évolution de l'âge au mariage mais pas toujours en phase avec ceux de la fécondité. En conséquence, si au niveau national comme au niveau régional la baisse de la fécondite est bien liée à la montée de l'âge au mariage des femmes, les liens entre les variations de la structure géographique de la fécondité et celles de la nuptialité sont plus complexes et ne laissent pas apparaître de correlation très évidente. Une des grandes difficultés à laquelle on se heurte pour cette mise en relation tient au fait que nous ne disposons pour suivre l'évolution de l'âge au mariage que des quelques mesures indirectes tirées des recensements décennaux et qui se rapportent à des périodes assez larges aux contours imprécis. Cela ne permet pas de coupler correctement les périodes d'évolution de la nuptialité à celles où la fécondité pourrait en subir l'inlfuence. De nouvelles analyses pourraient peutêtre être plus fructueuse lorsqu'il sera possible d'accéder aux données d'état civil sur le mariage qui permettraient de construire des tables de nuptialité annuelles par gouvernorat depuis les années 1960, que l'on pourrait confronter à l'évolution annuelle des TFT.

\section{Références}

ADLAKHA Arjun, AYAD Mohamed et KUMAR Sushil, 1991. - The role of nuptiality in fertility decline: a comparative analysis, in: Demographic and Health Surveys World Conference, Washington, August, 5-7 1991, (2): 947-964. - Columbia (Mar); IRD/Macro-International.

AlOUI Touhami, AYAD Mohamed et FouratI Habib (éd.), 1989. - Enquête démographique et de santé en Tunisie, 1988. - Tunis, Office national de la famille et de la population ; Columbia
(Maryland), IRD/Macro Systems Inc., 224 p. (DHS : Demographic and Health Survey)

AYAD Mohamed et JEMAI Yolande, 1991. - Fertility decline in Tunisia : factors affecting recent changes, in : James ALLMAN (ed.), Women's status and fertility in the Muslim world, p. 152163. - New York, Praeger publ., 378 p.

AYAD Mohamed et ZoughLAMI Younès, 1985. Fécondité et planification familiale en Tunisie, 1983. Rapport sur les résultats l'Enquête tunisienne sur la prévalence de la contraception. Tunis, ONFP ; Columbia (Mar), WPAS, I 76 p.

AYAD Mohamed et JEMAl Heidi, 200I. - Les déterminants de la fécondité, in : Jacques VALLIN et Thérèse $\mathrm{LOCOH}$ (dir), Population et développement en Tunisie : la métamorphose. p. I7I-20I. - Tunis, CERES, 80 I p.

BEN JELASSI Hechmi, 1992. - Différentielles de fécondité en Tunisie, in : Démographie et différences, Colloque international de Montréal, p. 16|-|68. - Paris, AIDELF, $662 \mathrm{p}$.

BEN RABAH, 201I. - Représenter l'évolution démographique en Tunisie (1975-2004) : quelles cartes dynamiques? M@ppemonde, 101 (2011.1), 17 pages. http://mappemonde.mgm.fr/num29/ articles/art I | |0 I.html

BENSALEM Lilia et LOCOH Thérèse, 200I. - Les transformations du mariage et de la famille, in : Jacques VALLIN et Thérèse LOCOH (dir.), Population et développement en Tunisie : la métamorphose, p. 153-172. - Tunis, Cérès Éditions, $801 \mathrm{p}$.

BOURAOUI Abdelhamid, 1992. - Variations régionales de fécondité en Tunisie, in : Démographie et différences, Colloque international de Montréal, p.8I-84. - Paris, AIDELF, 662 p.

BOURCHACHEN Jamal, 1995. - Contribution de la nuptialité et de la contraception à la baisse de la fécondité au Maroc. Revue marocaine de droit et d'économie du développement, (36): 45-6I.

HAJNAL John, 1953. - Age at marriage and proportion marrying. Populations Studies, 7 (2) II I-I 36.

INS, s.d. - Annuaire statistique de la Tunisie. Institut national de la statistique, Tunis : Vol. 20, |970-7I, 467 p. ; Vol. 2I, 1972 et 1973, 405 p. ; Vol. 23, 1976-1977, 365 p. ; Vol. 24, 1978-1979, 278 p. ; Vol. 25, année 1980, 29l p. ; Vol. 26, année I98I, 288 p. ; Vol. 27, année 1982, 295 p. ; Vol. 28, année 1983, 277 p. ; Vol. 36, années 1992-1993, 252 p. ; Vol. 39, année 1996, $26 \mathrm{I}$ p.

INS, 1973. - Enquête nationale démographique 1968-1969 : Goraa et Oued el Khatef. - Tunis, 
Institut national de la statistique, 120 p. (Études et enquêtes de l'INS. $N^{\circ} 5$ ).

INS, 1974. - Enquête nationale démographique 1968-1969 : mouvement de la population, naissances, décès, migration. - Tunis, Institut national de la statistique, 100 p. (Études et enquêtes de l'INS. Série Démographie).

INS, 1986. - Annuaire statistique de la Tunisie. Vol. 30, 1985. - Tunis, Institut national de la statistique, $273 \mathrm{p}$.

INS, 1990. - Annuaire statistique de la Tunisie. Vol. 33, 1989. - Tunis, Institut national de la statistique, 24I p.

INS, 1992. - Annuaire statistique de la Tunisie. Vol. 35, 1991. - Tunis, Institut national de la statistique, $246 \mathrm{p}$.

INS, 1995. - Annuaire statistique de la Tunisie. Vol. 37, 1994. - Tunis, Institut national de la statistique, $212 \mathrm{p}$.

INS, 1996. - Projections de la population 1995-2015. Vol. 2 : niveau régional. - Tunis, Institut national de la statistique, $25 \mathrm{I} \mathrm{p}$.

INS, 1998. - Annuaire statistique de la Tunisie. Vol. 40, 1997. - Institut national de la statistique, Tunis, $26 \mathrm{I} \mathrm{p}$.

INS, 2002. - Projections de population 1999-2029. Tunis, Institut national de la statistique, $194 \mathrm{p}$.

INS, 2013. - Annuaire statistique de la Tunisie. Vol. 55, 2008-I2. - Tunis, Institut national de la statistique, $357 \mathrm{p}$.

INS, 20I5. - Taqyim bayanet ihsaiyat el hala el madania, el waqui ouel afaq [Evaluation des données statistiques d'état civil, réalités et perspectives], présentation à l'atelier sur "Les systèmes d'enregistrement de l'état civil » organisé par l'INS, Tunis, 3 déc. 20I5, 15 diapos. Site web

http://www.ins.nat.tn/seminaires/civile/pres 03 ar.pd f consulté le I5 janvier 2016.

JEMAI Yolande et JEMAI Hedi, 1979. - Application of methods of measuring the impact of family planning programs on fertility: the case of Tunisia, in: The methodology of measuring the impact of family planning programs on fertility: problems and issues, p. 140-244. - New York, UN Department of International Economic and Social Affairs, $253 \mathrm{p}$.

JEMAI Yolande et JeMAI Hedi, 1982. - An improved application of methods of measuring the impact of family planning programs on fertility: the case of Tunisia 1956-1979, in: Evaluation of measuring the impact of family planning programs on fertility: sources of variances, p. 210-257. - New York, UN Department of International Economic and Social Affairs, $290 \mathrm{p}$.
LAPHAM Robert, 1970. - Family planning and fertility in Tunisia. Demography 7, (2): 24I-253

MDCI, INS, UNICEF, 20I3. - Tunisie. Suivi de la situation des enfants et des femmes. Enquête par grappes à indicateurs multiples. - Tunis, Ministère du développement et de la coopération internationale, Institut national de la statistique et Fonds des nations unies pour l'enfance, 210 pages. (Projet MICS 4, 20I I-20I2)

OUADAH-BEDIDI Zahia et VALLIN Jacques, 2000. Maghreb : la chute irrésistible de la fécondité. Population et Société (359): I-4.

OUADAH-BEDIDI, Zahia, VALLIN Jacques et BOUCHOUCHA Ibtihel, 2012. - La fécondité au Maghreb, nouvelle surprise. Population et Sociétés (486): I-4.

OUADAH-BEDIDI, Zahia, VALLIN Jacques et BOUCHOUCHA Ibtihel, 2012. - Unexpected developments in Maghrebian fertility. Population and Societies_(486): I-4.

ONFP, 1982. - Fécondité et contraception en milieu urbain : gouvernorat de Tunis. - Tunis, Office national de la famille et de la population, $69 \mathrm{p}$.

ONFP, 1985. - Enquête tunisienne sur la prévalence contraceptive, 1983. - Tunis, Office national de la famille et de la population, XV-I76 p. ONFP, 1985. - Enquête tunisienne sur la prévalence contraceptive, 1983. - Tunis, Office national de la famille et de la population, $176 \mathrm{p}$.

ONFP, 1997. - Enquête tunisienne sur la santé de la mère et de l'enfant. Rapport principal. - Tunis, ONFP, 248 p. (Projet PAPCHILD)

ONFP, 2002. - Enquête tunisienne sur la santé de la famille. Rapport principal. Tunis, Office national de la famille et de la population, 350 p. (Projet PAPFAM)

OUADAH-BEDIDI Zahia, 2004. - Baisse de la fécondité en Algérie : transition de développement ou transition de crise ? - Paris, Institut d'études politiques, $710 \mathrm{p}$.

SANDRON Frédéric, 1998. - Baisse de la fécondité en Tunisie. - Paris, CEPED, 55 p. (Les Dossiers du CEPED, $n^{\circ}$ 49)

SEKLANI Mahmoud, 1974. - La population de la Tunisie. - Paris, CICRED, $189 \mathrm{p}$.

TABUTIN Dominique, 1982 - Nuptiality and fertility in the Maghreb, in : Lado T. Ruzikha (ed.), Nuptiality and fertility. Proceedings of a seminar held in Bruges, 8-II January 1979. - Liège, IUSSP, Ordina Editions, $406 \mathrm{p}$.

VALLIN Jacques, 197I. - La nuptialité en Tunisie, Population, 26, (Special"Le Maghreb"): 250-266.

VALLIN Jacques et LAPHAM Robert J., 1969. - Place du planning familial dans l'évolution récente de la 
natalité en Tunisie, Revue tunisienne de sciences sociales, volume 6, p. 379-4I4

WALPERGER Dominique, VALLIN Jacques et BEN MRAD, 200I. - La dynamique naturelle de la population depuis l'indépendance, in : Jacques VALLIN et Thérèse LOCOH (dir.), Population et développement en Tunisie : la métamorphose, p. 53-88. - Tunis, Cérès Éditions, 801 p.

' "Ce travail a été réalisé dans le cadre du laboratoire d'excellence iPOPS porté par heSam Université, portant la référence ANR-I0-LABX-0089. Ce travail a bénéficié d'une aide de l'Etat gérée par l'Agence Nationale de la recherche au titre du projet Investissements d'Avenir Paris Nouveaux Mondes portant la référence $n^{\circ}$ ANR-I I-IDEX0006-02. »

ii La loi modifiant le code du statut personnel pour relever l'âge minimum légal au mariage des filles

à 17 ans (au lieu de 15 ans) a été promulguée le 20 février 1964. Cependant, durant toute l'année 1963 elle avait fait l'objet d'une large campagne d'information et de motivation de l'opinion publique. Cette campagne a eu pour effet secondaire un phénomène d'anticipation, les parents s'apprêtant à marier leur fille avant cet âge ont précipité son mariage afin qu'il ne puisse pas leur être refusé. Cela a provoqué un pic de mariages en 1963, suivi d'un pic de naissances en 1964 et même d'un second pic en 1966, dû à l'augmentation des naissances de rang 2 . C'est néanmoins cette loi de 1964 qui a été l'amorce de la baisse de la fécondité, car, passé cet épiphénomène d'anticipation, elle a durablement réduit le nombre de mariages précoces et donc la fécondité après 1964 car son effet a été, à ce stade, beaucoup plus marqué que celui de la contraception, le programme de planning familial n'en étant encore qu'à ses débuts (Vallin et Lapham, 1969, Vallin 197I).

iii Cette étude indique aussi que, contrairement à sa position affichée jusqu'ici, I'INS considère que l'enregistrement des naissances n'est pas encore tout à fait complet et propose des estimations corrigées du TFT pour les années 2004 à 2014. Aucune précision n'est cependant donnée sur les raisons et le comment de cette correction, qui au passage remettrait en cause le niveau de fécondité donné par l'enquête MICS-4 (alors même que précédemment les résultats des enquêtes spécialisées étaient toujours en accord avec les estimations reposant sur l'état civil et son taux de couverture supposé).

iv Béja, Bizerte, Gafsa, Gabès, Jendouba, Kairouan, Kasserine, Le Kef, Médenine, Nabeul, Sfax, Sousse, Tozeur, Tunis.

v Avec la création en 1973 de Sidi Bouzid (fait d'une partie de Gafsa à lauelle se sont ajoutées deux petites parties de Kasserine et de Sfax), en 1974 celles de Mahdia et de Monastir (détachés de Sousse), celle de Siliana (fait de parties du Kef et de Béja) et celle de Tunis-Sud constitué de parties de Tunis, Bizerte, Béja et Nabeul (Figure Ib).

vi En 1980 Tozeur est détaché de Gafsa, en 1981, Kébili est détaché de Gabes et Tataouine de Médenine, en 1983 l'Ariana est créé à partir d'une partie de Tunis et d'une partie de Zaghouan, tandis que Ben Arous est détaché de Tunis.

http://aps.journals.ac.za vii À la seule exception de la création d'Ariana, à partir d'un morceau de Tunis et d'un morceau de Zaghouan.

viii L'estimation de la population féminine célibataire par âge a été obtenue en faisant une interpolation linéaire entre les dates de recensements des proportions de célibataires entre les recensements et en les appliquant aux estimations annuelles de population de l'INS.

ix Ces deux gouvernorats n'existaient pas en 1975 mais on imagine facilement que l'AMPM n'a pas dû y augmenter beaucoup entre les recensements de 1975 et 1984 et qu'ils ont ensuite rattrapé leur retard. 